\title{
A role for nitric oxide in hydroxyurea-mediated fetal hemoglobin induction
}

\author{
S. Bruce King \\ Department of Chemistry, Wake Forest University, Winston-Salem, North Carolina, USA \\ J. Clin. Invest. 111:171-172 (2003). doi:10.1172/JCI200317597.
}

Hydroxyurea is a newly approved therapeutic agent for the treatment of sickle-cell disease. Hydroxyurea reduces the number of painful crises in sicklecell patients presumably by increasing the levels of fetal hemoglobin, which has a large solubilizing effect on sickle-cell hemoglobin and reduces polymerization (1). Despite being used to treat a number of cancers for nearly 30 years, the mechanism of how hydroxyurea increases fetal hemoglobin levels remains unclear. In this issue of the JCI, Cokic et al. present results that provide the first explanation of how hydroxyurea increases fetal hemoglobin levels (2). Specifically, they show that fetal hemoglobin increases in response to activation of soluble guanylyl cyclase (sGC) by hydroxyureaderived NO. The importance of this work manifests itself in at least three ways: 1$)$ the in vitro identification of some of the molecular species involved in fetal hemoglobin induction, 2) a further demonstration of a role for NO in the activity of hydroxyurea, and 3 ) the application of this work to the development of new NO based treatments for sickle-cell disease.

The work described in this study (2) relies on the authors' ability to recognize clues from previous basic and clinical research. First, hydroxyurea reacts with enzymes and proteins, particularly heme-containing proteins, to release

Address correspondence to: S. Bruce King, Department of Chemistry, Wake Forest University, Winston-Salem, North Carolina 27109, USA. Phone: (336) 758-5774; Fax: (336) 758-4656; E-mail: kingsb@wfu.edu. Conflict of interest: The author has declared that no conflict of interest exists.

Nonstandard abbreviations used: soluble guanylyl cyclase (sGC), S-nitrosocysteine (CysNO).
NO (3-7). Other studies reveal that patients taking hydroxyurea demonstrate increased levels of nitrite, nitrate, and iron nitrosyl hemoglobin, all of which are markers for NO (8-10). Finally, sGC activators increase $\gamma$-globin gene expression in both erythroleukemic cells and primary human erythroblasts and sGC inhibitors prevent this increase (11). The combination of these results with the ability of NO to stimulate sGC led to the design and execution of the described experiment to determine the effect of nitric oxide donors and hydroxyurea on fetal hemoglobin induction.

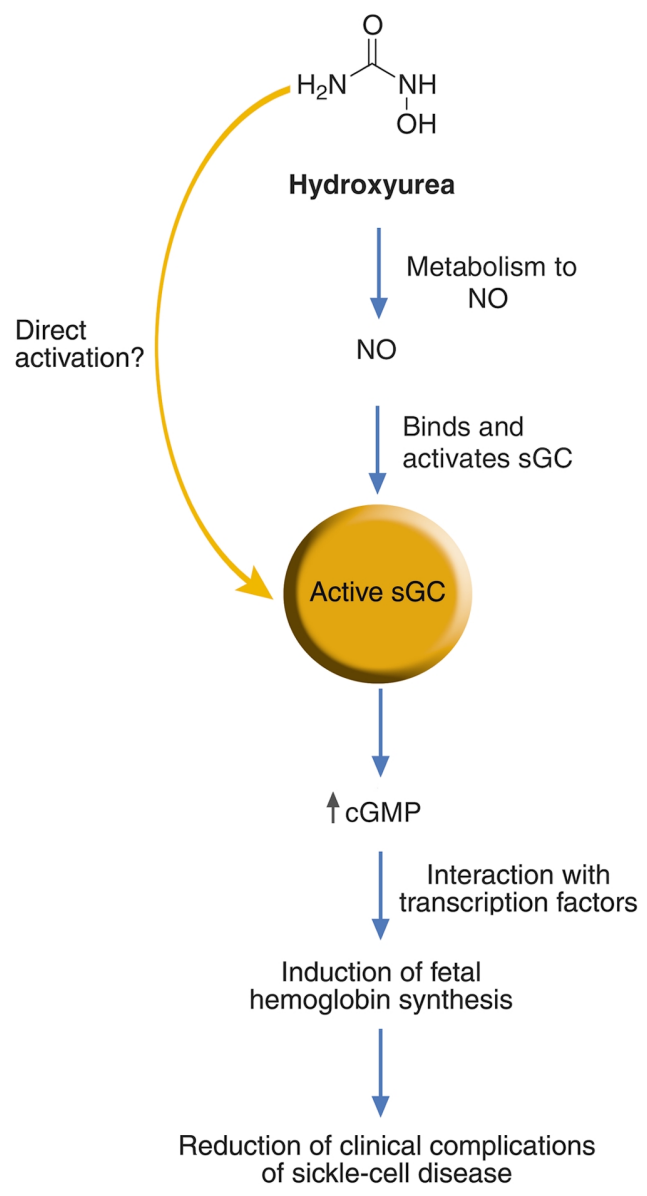

Figure 1

Mechanism of fetal hemoglobin induction by hydroxyurea. Hydroxyurea is metabolized by an unknown pathway to produce nitric oxide that binds and activates sGC. Alternatively, hydroxyurea may be able to directly activate sGC. Activation of sGC increases production of cGMP, which probably influences some transcription factors leading to increased fetal hemoglobin synthesis. 


\section{Hydroxyurea and NO donors increase $\gamma$-globin and cGMP levels}

Cokic et al. show that hydroxyurea and two other mechanistically different $\mathrm{NO}$ donors increase $\gamma$-globin gene expression in K562 erythroleukemic and human erythroid progenitor cells in a dose-dependent manner. These compounds also increase the $\gamma / \beta$ ratio in this system as they have little effect on $\beta$-globin gene expression. These cell-based assays, especially the human erythroid progenitor model, probably represent the best currently available systems to evaluate human responses to fetal hemoglobin-inducing agents. More importantly, hydroxyurea and S-nitrosocysteine (CysNO), a known NO donor, increase fetal hemoglobin levels in human erythroid cells and cGMP in erythroid progenitor cells. sGC inhibitors block the observed increase in $\gamma$-globin gene production. This last group of experiments provides strong evidence for the involvement of sGC in fetal hemoglobin induction and also reveals that hydroxyurea or NO donor-mediated $\gamma$-globin gene induction occurs only during a short period of time during erythroid differentiation.

\section{An NO-mediated pathway for fetal hemoglobin induction}

Figure 1 summarizes the current understanding of hydroxyurea-mediated induction of fetal hemoglobin synthesis. Based on the work of Cokic et al. (2) a link between hydroxyurea, NO, sGC, and fetal hemoglobin now exists (Figure 1). Figure 1 also shows where our understanding of this process remains incomplete, particularly: 1) how is hydroxyurea converted to $\mathrm{NO}$ in vivo, 2) can hydroxyurea directly activate sGC, and 3) how does an increase in cGMP induce fetal hemoglobin production? Chemically, the conversion of hydroxyurea to NO requires a three-electron oxidation. While a number of heme- and coppercontaining proteins react with hydroxyurea to produce NO in vitro through the intermediacy of both radical and nitroso intermediates (3-6), surprisingly little information exists regarding the in vivo oxidative metabolism of hydroxyurea (12). Some work suggests that NO release from hydroxyurea likely occurs during liver metabolism (13), but to date the actual site and oxidant of in vivo hydroxyurea metabolism to NO remains to be identified. Early work shows the ability of urease to catalyze the hydrolysis of hydroxyurea to hydroxylamine, which reacts rapidly with heme proteins to release NO (14). While such a route to NO should be explored, this pathway would be confined to areas of bacterial colonization, such as the gut, as no mammalian ureases exist. The authors present the idea that hydroxyurea may activate sGC through the direct reaction of the ferrous protein with hydroxyurea to produce the iron nitrosyl complex, in analogy to the reaction of hydroxyurea with deoxyhemoglobin, and this idea should also be examined (3). The authors present a number of reasonable but yet-to-be tested ideas involving the stimulation of fetal hemoglobin production by sGC activation (2). Mostly, these ideas elaborate on roles for transcription factors known to be controlled by the NO/cGMP pathway and require further experimentation to delineate the transcription factors responsible for fetal hemoglobin induction. The ability of hydroxyurea to inhibit ribonucleotide reductase and the possibility of this reaction to produce NO should also be further considered (15). At this time, other NOand sGC-independent pathways effected by hydroxyurea cannot be completely eliminated.

Finally, these results provide new insight into the development of NObased therapies for treatment of sicklecell disease. Inhaled NO and other NO donors, as well as strategies to increase endogenous NO production, should be strongly considered as sickle-cell therapies (16). Recent work by Cokic et al. also shows that the plasma of sicklecell patients contains cell-free hemoglobin that scavenges nitric oxide (17). The metabolism of hydroxyurea should be rigorously studied to identify the pathways that convert hydroxyurea to NO. The use of cGMP esterase inhibitors, which enhance the effects of NO on sGC, should also be viewed as an alternative approach for sickle-cell disease treatment. The results presented in this paper should excite the audience since they give both clinical and basic scientists a basis for rational therapeutic strategies for sickle-cell disease treatment. Further unraveling hydroxyurea's metabolism and the mechanism of cGMP-mediated fetal hemoglobin induction should lead to better strategies for sickle-cell disease therapy.

1. Charache, S. 1997. Mechanism of action of hydroxyurea in the management of sickle cell anemia in adults. Semin. Hematol. 34:15-21.

2. Cokic, V.P., et al. 2003. Hydroxyurea induces fetal hemoglobin by the nitric oxide-dependent activation of soluble guanylyl cyclase. J. Clin. Invest. 111:231-239. doi:10.1172/JCI200316672.

3. Huang, J., et al. 2002. Iron nitrosyl hemoglobin formation from the reactions of hemoglobin and hydroxyurea. Biochemistry. 41:2466-2474.

4. Huang, J., Sommers, E.M., Kim-Shapiro, D.B., and King, S.B. 2002. Horseradish peroxidase catalyzed nitric oxide formation from hydroxyurea. J. Am. Chem. Soc. 124:3473-3480.

5. Sato, K., et al. 1997. Nitric oxide generation from hydroxyurea via copper-catalyzed peroxidation and implications for pharmacological actions of hydroxyurea. Jpn. J. Cancer Res. 88:1199-1204.

6. Pacelli, R., Taira, J., Cook, J.A., Wink, D.A., and Krishna, M.C. 1996. Hydroxyurea reacts with heme proteins to generate nitric oxide. Lancet. 347:900

7. Stolze, K., and Nohl, H. 1990. EPR studies on the oxidation of hydroxyurea to paramagnetic compounds by oxyhemoglobins. Biochem. Pharmacol. 40:799-802.

8. Glover, R.E., Ivy, E.D., Orringer, E.P., Maeda, H., and Mason, R.P. 1999. Detection of nitrosyl hemoglobin in venous blood in the treatment of sickle cell anemia with hydroxyurea. Mol. Phar macology. 55:1006-1010.

9. Nahavandi, M., Wyche, M.Q., Perlin, E. Tavakkoli, F., and Castro, O. 2000. Nitric oxide metabolites in sickle cell anemia patients after oral administration of hydroxyurea: hemoglobinopathy. Hematol. 5:335-339.

10. Gladwin, M.T., et al. 2002. Nitric oxide donor properties of hydroxyurea in patients with sickle cell disease. Br. J. Hematol. 116:436-444.

11. Ikuta, T., Ausenda, S., and Cappellini, M.D. 2001 Mechanism for fetal globin gene expression: role of the soluble guanylate cyclase-cGMP-dependent protein kinase pathway. Proc. Natl. Acad. Sci. USA. 98:1847-1852.

12. Gwilt, P.R., and Tracewell, W.G. 1998. Pharmacokinetics and pharmacodynamics of hydroxyurea. Clin. Pharmacokinet. 34:347-356.

13. Jiang, J., et al. 1997. In vivo production of nitric oxide in rats after administration of hydroxyurea. Mol. Pharmacology. 52:1081-1086.

14. Fishbein, W.N., and Carbone, P.P. 1963. Hydroxyurea: mechanism of action. Science. 142:1069-1070.

15. Yarbro, J.W. 1992. Mechanism of action of hydroxyurea. Semin. Oncol. 19:1-10.

16. Gladwin, M.T., and Schechter, A.N. 2001. Nitric oxide therapy in sickle cell disease. Semin. Hematol. 38:333-342.

17. Reiter, C.D., et al. 2002. Cell-free hemoglobin limits nitric oxide bioavailability in sickle-cell disease. Nat. Med. 8:1383-1389. 